\title{
La mort et l'égalité en France
}

\section{Jean Martin}

Dr med., membre de la rédaction

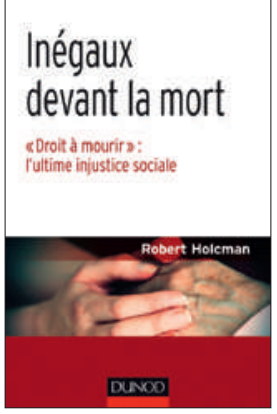

Robert Holcman Inégaux devant la mort

«Droit à mourir»: l'ultime injustice sociale

Paris: Dunod; 2015. 200 pages. $28.00 \mathrm{CHF}$. ISBN 978-2-100-72883-1
Economiste et gestionnaire français, ayant assumé des tâches dirigeantes en hôpital, enseignant, Robert Holcman publie un ouvrage dense sur l'éventail des facettes du thème "fin de vie et droit à mourir» qui fait beaucoup débat aujourd'hui. On y trouve la matière de beaucoup de prises de position et de réflexions sur ce sujet, ce qui peut faire de ce livre une référence utile. Une référence toutefois que salueront surtout ceux qui restent très réticents, voire fondamentalement opposés à la libre détermination des personnes dans ce domaine.

Une chose frappe dans le panorama brossé par l'auteur: on n'y trouve pas de récits cliniques, d'histoires de patients qui fassent toucher du doigt ce que vivent, en France aujourd'hui, les malades en fin de vie, leurs proches et les soignants. On est surtout confronté à des argumentations intellectuelles. Bien sûr, il importe de rappeler ce que les sociologues, les philosophes ou des instances officielles ont dit sur ces sujets. Mais il faudrait faire la part de ce qui est options dogmatiques, parfois répétées encore et encore, et de ce qu'on sait pratiquement. Le rapport de la Commission Sicard présenté en décembre 2012 a été limpide dans sa conclusion: «en général, on meurt mal en France aujourd'hui et il est urgent d'améliorer la situation». Pour cela, le développement des soins palliatifs est à l'évidence une avenue majeure mais ne saurait être l'entier de la solution. Au-delà, trois ans plus tard, Inégaux devant la mort ne suggère pas d'avancée notable; l'auteur dit même à plusieurs reprises ses réserves à l'endroit de la révision récente de la loi française dite Claeys Leonetti qui admet la sédation terminale.

Point d'importance: s'agissant d'inégalité, l'auteur rappelle à juste titre la constante, qui préoccupe fondamentalement les professionnels de santé publique, des inégalités dans l'accès aux soins pour les groupes moins favorisés, leur plus grande morbidité et leur moindre espérance de vie. Il craint que, dans la foulée, les personnes précarisées soient poussées plus que d'autres à envisager de mettre fin à leurs jours - cas échéant en y étant incités par leurs proches pour des motifs matériels. Préoccupation tout à fait estimable. Toutefois, si ce souci se comprend bien dans un pays comme les Etats-Unis où l'accès aux soins reste fort inégalitaire, c'est moins le cas en France où un système de santé social et étoffé doit assurer à tous une prise en charge adéquate.

Clairement, la grande différence entre Holcman et d'autres (dont le rédacteur de cette recension) est dans l'importance et le respect accordé à l'autonomie de la personne. Il y a dans le livre une réticence palpable à admettre que les patients ont le droit de décider de leur propre existence, y compris quant à l'option de lui mettre un terme quand elle est devenue trop lourde à porter. On peut, on doit bien sûr avoir le souci que ces décisions soient bien réfléchies mais cela ne saurait justifier le retour à une posture paternaliste, élitiste. Parlant d'enjeux de santé, de maladie et d'éthique, on ne peut accepter des raisonnements répétés tendant à disqualifier les patients qui ne pensent pas comme soi, dont l'avis serait forcément peu crédible s'ils sollicitent une aide au suicide. En 2015, près de quinze ans après la loi Kouchner sur les droits des malades, il y a là un anachronisme grave, ou de la cécité.

Il y a dans le livre une réticence palpable à admettre que les patients ont le droit de décider de leur propre existence.

A propos du titre du livre: il y a une inégalité, différente de celle qu'argumente l'auteur, qui au plan citoyen n'est pas admissible: la réalité qu'un statut social plus élevé facilite pour qui le souhaite d'avoir accès aux moyens de terminer sa vie. Qu'il suffise de rappeler que Mme Jospin, mère d'un premier ministre, a pu bénéficier d'une aide au suicide - qui n'a guère suscité de réprobation sociétale. N’y a-t-il pas lieu, au pays des droits de l'homme, de s'émouvoir de tels écarts, au détriment de la libre détermination des moins influents? A cet égard, Holcman évoque le fait indiscuté qu'il y a, en France et ailleurs, un nombre notable d'assistances au suicide et d'euthanasies qui ne disent pas leur nom. Il les regrette mais semble s'accommoder de cette clandestinité. A notre sens, le fait choquant que ces choses soient tolérées sous le manteau enlève beaucoup au poids d'argumentations dogmatiques refusant de considérer dans la clarté une évolution sociétale profonde. 